Article

\title{
Bisphenol A Release from Dental Composites and Resin-Modified Glass Ionomers under Two Polymerization Conditions
}

\author{
Antonin Tichy ${ }^{1, *(\mathbb{D})}$, Marketa Simkova ${ }^{2,3, * \mathbb{D}}$, Radka Vrbova ${ }^{1}$, Adela Roubickova ${ }^{1}$, Michaela Duskova ${ }^{2}$ \\ and Pavel Bradna ${ }^{1}$ \\ 1 Institute of Dental Medicine, First Faculty of Medicine, Charles University, General University Hospital, \\ Karlovo Namesti 32, 12111 Prague, Czech Republic; radka.vrbova@lf1.cuni.cz (R.V.); \\ adela.roubickova@lf1.cuni.cz (A.R.); pavel.bradna@lf1.cuni.cz (P.B.) \\ 2 Institute of Endocrinology, Narodni 8, 11694 Prague, Czech Republic; mduskova@endo.cz \\ 3 Department of Natural Compounds, University of Chemistry and Technology, Technicka 6, \\ 16628 Prague, Czech Republic \\ * Correspondence: antonin.tichy@lf1.cuni.cz (A.T.); msimkova@endo.cz (M.S.)
}

\section{check for}

updates

Citation: Tichy, A.; Simkova, M.; Vrbova, R.; Roubickova, A.; Duskova, M.; Bradna, P. Bisphenol A Release from Dental Composites and Resin-Modified Glass Ionomers under Two Polymerization Conditions. Polymers 2022, 14, 46. https://doi.org/10.3390/polym 14010046

Academic Editors: Eija Säilynoja, Sufyan Garoushi and Lippo Lassila

Received: 30 November 2021

Accepted: 21 December 2021

Published: 23 December 2021

Publisher's Note: MDPI stays neutral with regard to jurisdictional claims in published maps and institutional affiliations.

Copyright: (C) 2021 by the authors. Licensee MDPI, Basel, Switzerland. This article is an open access article distributed under the terms and conditions of the Creative Commons Attribution (CC BY) license (https:// creativecommons.org/licenses/by/ $4.0 /)$.

\begin{abstract}
Bisphenol A (BPA)-based monomers are commonly contained in dental resin-based materials. As BPA is an endocrine disruptor, its long-term release from restorative composites and resin-modified glass ionomers (RM-GICs) under two polymerization conditions was measured in this study. Specimens of two conventional composites containing BPA-based monomers, two "BPA-free" composites, and two RM-GICs were polymerized from one side for $20 \mathrm{~s}$ at $1300 \mathrm{~mW} / \mathrm{cm}^{2}$ or for $5 \mathrm{~s}$ at $3000 \mathrm{~mW} / \mathrm{cm}^{2}$. The amounts of BPA released in artificial saliva and methanol after 1, 4, 9, 16, 35, 65, 130, and 260 days were measured using liquid chromatography-tandem mass spectrometry. The highest amounts of BPA were released from conventional composites, followed by RM-GICs, while the least was released from "BPA-free" composites. Amounts of released BPA were significantly higher in methanol and decreased gradually after the first day. Fast polymerization $\left(5 \mathrm{~s}\right.$ at $\left.3000 \mathrm{~mW} / \mathrm{cm}^{2}\right)$ resulted in a significantly higher release of BPA after 1 day, but the effect of polymerization conditions was not significant overall. In conclusion, fast polymerization increased the initial release of BPA, but the released amounts were significantly lower than the current tolerable daily intake $(4 \mu \mathrm{g} / \mathrm{kg}$ body weight/day) even in methanol, representing the worst-case scenario of BPA release.
\end{abstract}

Keywords: bisphenol A; Bis-GMA; resin composite; glass ionomer cements; light-curing; liquid chromatography; mass spectrometry

\section{Introduction}

Due to its structural similarity with some hormones, bisphenol A (2,2-bis (4-hydroxyphenyl)propane, BPA) is able to bind to various receptors [1,2] and acts as an endocrine disruptor [3]. As a consequence, it has been associated with reproductive, developmental, metabolic, and other disorders [4,5]. However, exposure to BPA is surrounded by controversy. While the U.S. Food and Drug Administration (FDA) and the European Food Safety Authority (EFSA) declared BPA safe at current exposure levels (estimated to be $1.449 \mu \mathrm{g} / \mathrm{kg}$ body weight/day) [6,7], some authors have suggested that adverse effects may occur at doses much below the tolerable daily intake (TDI) [8-11] of $4 \mu \mathrm{g} / \mathrm{kg}$ b.w. set by the EFSA in 2015 [7].

The use of BPA-based polymers is widespread in food-contact materials and many other products, including dental materials containing BPA-based monomers [12,13], such as bisphenol A glycidyl dimethacrylate (Bis-GMA). As BPA is not intentionally added to dental materials, it is usually present as an impurity from the synthesis of BPA-based monomers or a product of their hydrolytic degradation [14]. Concerns [15] over the release of BPA from dental materials emerged in 1996 after high levels of BPA were detected in 
the saliva of patients treated with a sealant containing bisphenol A dimethacrylate (BisDMA) [16]. These results were disputed $[17,18]$ and numerous following studies reported that the release of BPA from dental materials was significantly lower than TDI [19,20], which led the Scientific Committee on Emerging and Newly Identified Health Risks (SCENIHR) to the conclusion that long-term oral exposure to BPA via dental materials poses only a negligible risk to human health [21]. Nevertheless, due to the apprehension of the lowdose effect [8-11], BPA-based monomers were excluded from some composites labeled as "BPA-free."

The release of monomers and other components from resin composites and other resin-based restorative materials, such as resin-modified glass ionomer cements (RMGICs), is known to be inversely proportional to the degree of conversion [22]. However, the effect of polymerization conditions on the release of BPA has not been sufficiently examined. Manabe et al. reported that the release of BPA from uncured fissure sealants and resin composites in phosphate-buffered saline was significantly higher than if they were polymerized for $60 \mathrm{~s}$ [23]. On the other hand, Kwon et al. found that the release of BPA from four resin composites increased with extended irradiation time and reduced distance between the lamp tip and composite surface [24], which was attributed to the photolysis of BPA-based monomers under the high intensity of polymerization light [24]. The effect of irradiation time was also studied by Polydorou et al., but the results were inconclusive as no BPA was detected in most extracts [25]. These ambiguous results suggested that sensitive detection methods are necessary for the trace analysis of released BPA. While the lower limit of quantification (LLOQ) of the liquid chromatography-tandem mass spectrometry (LC-MS/MS) method used by Polydorou et al. was $500 \mathrm{ng} / \mathrm{mL}$ [25], the current ultra-highperformance LC-MS/MS (UPLC-MS/MS) methods combined with BPA derivatization reached a LLOQ below $0.1 \mathrm{ng} / \mathrm{mL}$ [26-28].

The shortening of restorative procedures is one of the targets of contemporary dental material research, and the recent development of high-power polymerization lamps enabled the reduction in irradiation time from $20 \mathrm{~s}$ to less than $10 \mathrm{~s}$. Therefore, the objective of this study was to compare the effect of fast polymerization, i.e., irradiation for $5 \mathrm{~s}$ at high irradiance, and standard polymerization, i.e., irradiation for $20 \mathrm{~s}$ at moderate irradiance, on the long-term release of BPA from various restorative materials measured using an UPLC-MS/MS method with dansyl chloride derivatization [26]. Tested materials included composites containing BPA-based monomers, hereinafter referred to as conventional composites, "BPA-free" composites, and RM-GICs, in which BPA-based monomers were identified in previous studies [29,30]. Artificial saliva, representing the oral environment, and methanol, simulating the worst-case scenario of BPA release, were selected as the extraction media. The null hypotheses tested were (1) that there would be no difference in the release of BPA between fast and standard polymerization, and (2) that there would be no difference in the release of BPA between the tested materials.

\section{Materials and Methods}

Conventional composites Charisma Classic (CC; Kulzer, Hanau, Germany) and Filtek Ultimate Universal Restorative (FU; 3M, St. Paul, MN, USA), "BPA-free" composites Charisma Diamond (CD; Kulzer, Hanau, Germany) and Admira Fusion (AF; Voco, Cuxhaven, Germany), and RM-GICs Photac Fil Quick Aplicap (PF; 3M) and GC Fuji II LC Capsule (F2; GC, Tokyo, Japan) were investigated. According to the material safety data sheets, CC contains Bis-GMA, while FU contains Bis-GMA and ethoxylated Bis-GMA (Bis-EMA). The presence of BPA-based monomers in the examined "BPA-free" composites and RMGICs was not officially disclosed; however, previous studies identified Bis-EMA in PF [29] and Bis-GMA in F2 $[29,30]$. The composition of the materials is summarized in Table 1. 
Table 1. Composition of materials tested in this study.

\begin{tabular}{ccc}
\hline Material (Abbreviation) & Manufacturer (Batch Number) & Composition \\
\hline Charisma Classic A2 (CC) & Kulzer, Hanau, Germany (K010733) & $\begin{array}{c}\text { Bis-GMA, TEGDMA, Ba-Al-F glass fillers, } \\
\text { pre-polymerized filler, pyrogenic } \\
\text { silica, initiator }\end{array}$ \\
$\begin{array}{c}\text { Filtek Ultimate Universal Restorative A2 } \\
\text { Dentin (FU) }\end{array}$ & 3M, St. Paul, MN, USA (N985020) & $\begin{array}{c}\text { Bis-GMA, Bis-EMA, } \\
\text { UDMA, TEGDMA, PEGDMA, } \\
\text { and zirconia filler, aggregated } \\
\text { zirconia/silica cluster filler, initiator }\end{array}$ \\
\hline Charisma Diamond A2 (CD) & Kulzer, Hanau, Germany (K010073) & $\begin{array}{c}\text { TCD-DI-HEA, UDMA, TEGDMA, } \\
\text { Ba-Al-F glass fillers, pyrogenic } \\
\text { silica, initiator }\end{array}$ \\
\hline
\end{tabular}

Admira Fusion A2 (AF)

Voco, Cuxhaven, Germany (1919447)

Photac Fil Quick Aplicap A2 (PF)

3M, St. Paul, MN, USA (4587570) no conventional methacrylate monomers, "organically modified ceramics" resin, glass ceramic filler, nano filler, initiator

Na-Ca-Al-La-F silicate glass, HEMA, difunctional monomers, activator (amine), copolymer of acrylic acid and maleic acid, camphorquinone; stabilizers

Al-F silicate glass, polyacrylic acid, HEMA, 2,2,4-trimethyl hexamethylene dicarbonate, TEGDMA

GC Fuji II LC Capsule A2 (F2)

GC, Tokyo, Japan (190219A)

dicarbc

Abbreviations: Bis-GMA-bisphenol A glycidyl dimethacrylate; TEGDMA—triethylene glycol dimethacrylate; Bis-EMA-ethoxylated bisphenol A glycol dimethacrylate; UDMA-urethane dimethacrylate; PEGDM-polyethylene glycol dimethacrylate; TCD-DI-HEA-bis-(acryloyloxymethyl) tricyclodecane; HEMA-2-hydroxyethyl methacrylate.

\subsection{Specimen Preparation and Extraction}

Polytetrafluoroethylene (PTFE) molds (6 mm diameter, $2 \mathrm{~mm}$ thickness) placed on a glass slide were filled with the materials and their surface was flattened using another glass slide. The specimens (surface area $94.2 \mathrm{~mm}^{2}$, volume $56.5 \mathrm{~mm}^{3}$, mass $0.13-0.16 \mathrm{~g}$ ) were polymerized from one side using the Valo LED polymerization lamp (Ultradent Products, South Jordan, UT, USA) either for $20 \mathrm{~s}$ in "standard" mode (irradiance $1300 \mathrm{~mW} / \mathrm{cm}^{2}$ ) or for $5 \mathrm{~s}$ in "plasma emulation" mode $\left(3000 \mathrm{~mW} / \mathrm{cm}^{2}\right)$, which corresponds to radiant exposures of $26 \mathrm{~J} / \mathrm{cm}^{2}$ and $15 \mathrm{~J} / \mathrm{cm}^{2}$, respectively. The irradiance in each mode was measured through a glass slide using a USB2000+ spectrometer connected via an optical fiber with a CC-3 cosine corrector. Five measurements per mode were performed after the measuring apparatus had been calibrated with a traceable light source HL-3P-CAL (all Ocean Optics, Dunedin, FL, USA) [31]. The data were processed using SpectraSuite Ocean Optics software.

Thirty minutes after polymerization, specimens were weighed using a digital analytical balance accurate to $0.1 \mathrm{mg}$ and transported to borosilicate glass test tubes with $2 \mathrm{~mL}$ of artificial saliva or LC-MS-grade methanol $(n=3)$. The artificial saliva (Hospital laboratory; General University Hospital in Prague, Czech Republic) was prepared by dissolving $0.8 \mathrm{~g} / \mathrm{L}$ of $\mathrm{NaCl}, 1.2 \mathrm{~g} / \mathrm{L}$ of KCl, $0.1 \mathrm{~g} / \mathrm{L}$ of $\mathrm{CaCl}_{2} \cdot 2 \mathrm{H}_{2} \mathrm{O}, 0.3 \mathrm{~g} / \mathrm{L}$ of $\mathrm{K}_{2} \mathrm{HPO}_{4} \cdot 3 \mathrm{H}_{2} \mathrm{O}$, and $0.1 \mathrm{~g} / \mathrm{L}$ of $\mathrm{MgCl}_{2} \cdot 6 \mathrm{H}_{2} \mathrm{O}$ in distilled water [32] with the $\mathrm{pH}$ adjusted to 7.0. The test tubes were closed using screw caps with PTFE-faced septa and incubated at $37^{\circ} \mathrm{C}$ in darkness with daily manual agitation. Extracts were collected after 1, 4, 9, 16, 35, 65, 130, and 260 days. When refreshing the extraction media, the test tubes were rinsed 5 times with $0.5 \mathrm{~mL}$ of the respective extraction medium, and specimens were carefully removed, weighed using the analytical balance, and replaced in test tubes with $2 \mathrm{~mL}$ of fresh extraction medium. To prevent contamination, all instruments, test tubes, and molds had been repeatedly rinsed with methanol. 


\subsection{Uptake of Extraction Media and Mass Loss}

After the last extract was collected, the specimens were weighed, dried at room temperature, and repeatedly weighed until a constant mass was obtained. Based on the mass after specimen polymerization $\left(m_{1}\right)$, the highest mass measured during the extraction period $\left(m_{2}\right)$, and the final mass in the dry state $\left(m_{3}\right)$, the uptake of artificial saliva and methanol was calculated as $\left(m_{2}-m_{3}\right) / m_{1}$. Mass loss was calculated using the equation $\left(m_{1}-m_{3}\right) / m_{1}$. The uptake and mass loss were not calculated for RM-GICs, because they inherently contain water, and the results could therefore be misleading.

\subsection{Chromatographic Analysis}

For the LC-MS/MS analysis, BPA and deuterated BPA (d16BPA) standards, dansyl chloride, acetone, sodium bicarbonate, and ammonium formate were purchased from Sigma-Aldrich (St. Louis, MO, USA). LC-MS grade methanol, water for chromatography, and diethylether were obtained from Merck AG (Darmstadt, Germany). Methanol p.a. was purchased from Lach-Ner (Neratovice, Czech Republic). Following the protocol of our previous study [26], a nine-point calibration curve was prepared using $0.032-8.0 \mathrm{ng} / \mathrm{mL}$ solutions of BPA in methanol. An amount of $10 \mu \mathrm{L}$ of the internal standard (d16BPA in methanol) was added to $700 \mu \mathrm{L}$ of each extract, artificial saliva was extracted using diethylether, and all samples were dried under reduced pressure. The same protocol was used for blanks and control samples with a known addition of BPA. The derivatization of BPA was performed according to [2,33]. Dry residues of the samples were vortexed with $50 \mu \mathrm{L}$ of dansyl chloride in acetone $(1 \mathrm{mg} / \mathrm{mL})$ and $50 \mu \mathrm{L}$ of a $100 \mathrm{mM}$ sodium bicarbonate buffer, incubated at $50{ }^{\circ} \mathrm{C}$ for $15 \mathrm{~min}$ and evaporated to dryness. Then, $300 \mu \mathrm{L}$ of methanol were added and equally diluted with a $10 \mathrm{mM}$ aqueous solution of ammonium formate. An amount of $50 \mu \mathrm{L}$ of the solution was used for the LC-MS/MS analysis, which was performed using an API 3200 (Sciex, Concord, Canada), a triple-stage quadrupole mass spectrometer with electrospray ionization (ESI) connected to the Eksigent ultra-LC 110 system (Redwood City, CA, USA). Chromatographic separation was performed using a Kinetex C18 $1.7 \mu \mathrm{m}(150 \times 3.0 \mathrm{~mm})$ column (Phenomenex, Torrance, CA, USA) equipped with a security guard at a flow rate of $0.4 \mathrm{~mL} / \mathrm{min}$ at $50{ }^{\circ} \mathrm{C}$. A mixture of methanol and water was used as the mobile phase. Further information about LC-MS/MS conditions is available in [2,33]. The LLOQ was $0.042 \mathrm{ng} / \mathrm{mL}$.

\subsection{Statistical Analysis}

The measured amounts of BPA were divided by the specimen mass $\left(m_{1}\right)$ to obtain amounts of BPA released per gram of material $(\mathrm{ng} / \mathrm{g})$. To allow for the analysis of the kinetics of BPA release, the average daily release was calculated by dividing the amount of BPA in the extract by the extraction time in days. As eight extracts were prepared from each specimen, the average daily release of BPA from each material in the artificial saliva/methanol was analyzed using two-way repeated measures ANOVA (factors polymerization conditions and extraction time), and pairwise comparisons were performed using Fisher's LSD test. Cumulative amounts of BPA released from each material over the entire period of 260 days were analyzed using two-way ANOVA (factors extraction medium and polymerization conditions), and materials were compared using t-tests. The uptake of extraction media and mass loss of each tested composite were analyzed analogously to cumulative amounts of released BPA. All analyses were performed at a significance level of 0.05 using Statistica software (version 12.0, TIBCO, Palo Alto, CA, USA).

\section{Results}

\subsection{Release of $B P A$}

The average daily releases of BPA in the artificial saliva and methanol are presented in Tables 2 and 3, respectively. The kinetics of the release and differences caused by polymerization conditions and extraction media are illustrated in Figure 1. In both extraction media, all materials released the highest amounts of BPA during the first day. The release 
decreased significantly in the following days $(p<0.001)$ and continued over the entire 260 day period, gradually approaching zero from "BPA-free" composites and the RM-GIC F2. In contrast, the release of BPA from PF in methanol reached its minimum after 35 days, and then it increased slightly.

Average daily release of BPA
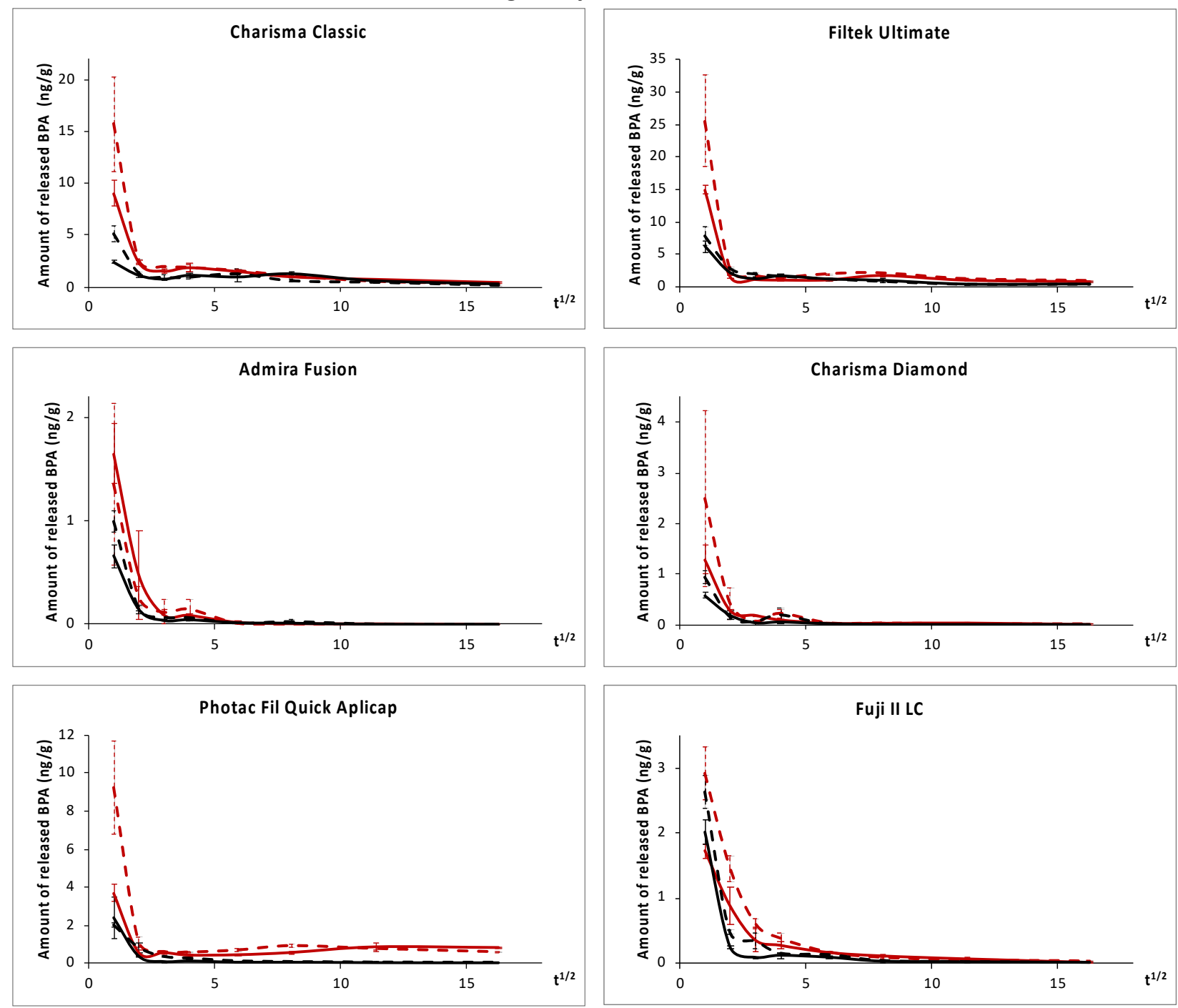

_Methanol (20s) _ - Methanol (5s)

Artificial saliva (20 s)

- - Artificial saliva (5 s)

Figure 1. Average daily release of BPA in the artificial saliva (black) and methanol (red) plotted against the square root of time. The solid lines represent standard polymerization (20 s, $\left.1300 \mathrm{~mW} / \mathrm{cm}^{2}\right)$, whereas dashed lines represent fast polymerization $\left(5 \mathrm{~s}, 3000 \mathrm{~mW} / \mathrm{cm}^{2}\right)$. 
Table 2. Average daily release of BPA in the artificial saliva per gram of material (mean $\pm \mathrm{SD}$ in $\mathrm{ng} / \mathrm{g} /$ day).

\begin{tabular}{|c|c|c|c|c|c|c|c|c|c|}
\hline Material & Polymer Conditions & 1 Day (Day 1) & $\begin{array}{l}4 \text { Days } \\
\text { (Days 2-4) }\end{array}$ & $\begin{array}{l}9 \text { Days } \\
\text { (Days 5-9) }\end{array}$ & $\begin{array}{c}16 \text { Days } \\
\text { (Days 10-16) }\end{array}$ & $\begin{array}{c}35 \text { Days } \\
\text { (Days 17-35) }\end{array}$ & $\begin{array}{c}65 \text { Days } \\
\text { (Days 36-65) }\end{array}$ & $\begin{array}{c}130 \text { Days } \\
\text { (Days 66-130) }\end{array}$ & $\begin{array}{c}260 \text { Days } \\
\text { (Days 130-260) }\end{array}$ \\
\hline \multirow{2}{*}{$\begin{array}{l}\text { Charisma } \\
\text { Classic }\end{array}$} & $20 \mathrm{~s}, 1300 \mathrm{~mW} / \mathrm{cm}^{2}$ & $2.43 \pm 0.19 \mathrm{Aa}$ & $1.11 \pm 0.12 \mathrm{Abc}$ & $0.73 \pm 0.02 \mathrm{Acd}$ & $1.11 \pm 0.20 \mathrm{Abc}$ & $\begin{array}{c}0.95 \pm 0.38 \\
\text { Abcd }\end{array}$ & $1.27 \pm 0.19 \mathrm{Bb}$ & $0.57 \pm 0.05$ Ade & $0.25 \pm 0.04 \mathrm{Ae}$ \\
\hline & $5 \mathrm{~s}, 3000 \mathrm{~mW} / \mathrm{cm}^{2}$ & $5.09 \pm 0.79 \mathrm{Ba}$ & $1.36 \pm 0.11 \mathrm{Ab}$ & $0.97 \pm 0.19 \mathrm{Abc}$ & $0.99 \pm 0.18 \mathrm{Abc}$ & $1.31 \pm 0.40 \mathrm{Ab}$ & $0.64 \pm 0.09 \mathrm{Acd}$ & $0.50 \pm 0.10 \mathrm{Acd}$ & $0.21 \pm 0.03 \mathrm{Ad}$ \\
\hline \multirow{2}{*}{$\begin{array}{c}\text { Filtek } \\
\text { Ultimate } \\
\text { Universal } \\
\text { Restorative }\end{array}$} & $20 \mathrm{~s}, 1300 \mathrm{~mW} / \mathrm{cm}^{2}$ & $6.24 \pm 0.84 \mathrm{Aa}$ & $2.23 \pm 0.09 \mathrm{Ab}$ & $1.28 \pm 0.12 \mathrm{Acd}$ & $1.71 \pm 0.31 \mathrm{Abc}$ & $1.25 \pm 0.14 \mathrm{Acd}$ & $1.03 \pm 0.39 \mathrm{Acd}$ & $0.46 \pm 0.07 \mathrm{Ad}$ & $0.48 \pm 0.27 \mathrm{Ad}$ \\
\hline & $5 \mathrm{~s}, 3000 \mathrm{~mW} / \mathrm{cm}^{2}$ & $7.87 \pm 1.37 \mathrm{Ba}$ & $2.82 \pm 0.10 \mathrm{Bb}$ & $1.95 \pm 0.26 \mathrm{Ac}$ & $1.62 \pm 0.14 \mathrm{Acd}$ & $1.18 \pm 0.18$ Acd & $0.80 \pm 0.21$ Ade & $0.35 \pm 0.04 \mathrm{Ae}$ & $0.35 \pm 0.03 \mathrm{Ae}$ \\
\hline \multirow{2}{*}{$\begin{array}{l}\text { Charisma } \\
\text { Diamond }\end{array}$} & $20 \mathrm{~s}, 1300 \mathrm{~mW} / \mathrm{cm}^{2}$ & $0.58 \pm 0.06 \mathrm{Aa}$ & $0.19 \pm 0.08 \mathrm{Ab}$ & $0.03 \pm 0.03 \mathrm{Ac}$ & $0.05 \pm 0.01 \mathrm{Ac}$ & $0.02 \pm 0.00 \mathrm{Ac}$ & $0.00 \pm 0.00 \mathrm{Ac}$ & $0.00 \pm 0.00 \mathrm{Ac}$ & $0.00 \pm 0.00 \mathrm{Ac}$ \\
\hline & $5 \mathrm{~s}, 3000 \mathrm{~mW} / \mathrm{cm}^{2}$ & $0.93 \pm 0.12 \mathrm{Ba}$ & $0.15 \pm 0.06 \mathrm{Ab}$ & $0.07 \pm 0.01 \mathrm{Abc}$ & $0.10 \pm 0.01 \mathrm{Abc}$ & $0.01 \pm 0.00 \mathrm{Ac}$ & $0.01 \pm 0.01 \mathrm{Ac}$ & $0.00 \pm 0.00 \mathrm{Ac}$ & $0.00 \pm 0.00 \mathrm{Ac}$ \\
\hline \multirow{2}{*}{$\begin{array}{l}\text { Admira } \\
\text { Fusion }\end{array}$} & $20 \mathrm{~s}, 1300 \mathrm{~mW} / \mathrm{cm}^{2}$ & $0.66 \pm 0.11 \mathrm{Aa}$ & $0.14 \pm 0.04 \mathrm{Ab}$ & $0.04 \pm 0.01 \mathrm{Ac}$ & $0.04 \pm 0.01 \mathrm{Ac}$ & $0.02 \pm 0.00 \mathrm{Ac}$ & $0.01 \pm 0.01 \mathrm{Ac}$ & $0.00 \pm 0.00 \mathrm{Ac}$ & $0.00 \pm 0.00 \mathrm{Ac}$ \\
\hline & $5 \mathrm{~s}, 3000 \mathrm{~mW} / \mathrm{cm}^{2}$ & $1.00 \pm 0.10 \mathrm{Ba}$ & $0.15 \pm 0.02 \mathrm{Ab}$ & $0.07 \pm 0.02 \mathrm{Ac}$ & $0.06 \pm 0.03 \mathrm{Ac}$ & $0.01 \pm 0.00 \mathrm{Ac}$ & $0.03 \pm 0.02 \mathrm{Ac}$ & $0.00 \pm 0.00 \mathrm{Ac}$ & $0.00 \pm 0.00 \mathrm{Ac}$ \\
\hline \multirow{2}{*}{$\begin{array}{c}\text { Photac Fil } \\
\text { Quick Aplicap }\end{array}$} & $20 \mathrm{~s}, 1300 \mathrm{~mW} / \mathrm{cm}^{2}$ & $2.40 \pm 1.07 \mathrm{Aa}$ & $0.37 \pm 0.04 \mathrm{Ab}$ & $0.09 \pm 0.01 \mathrm{Ab}$ & $0.13 \pm 0.02 \mathrm{Ab}$ & $0.06 \pm 0.01 \mathrm{Ab}$ & $0.06 \pm 0.02 \mathrm{Ab}$ & $0.03 \pm 0.00 \mathrm{Ab}$ & $0.03 \pm 0.00 \mathrm{Ab}$ \\
\hline & $5 \mathrm{~s}, 3000 \mathrm{~mW} / \mathrm{cm}^{2}$ & $2.02 \pm 0.12 \mathrm{Aa}$ & $0.84 \pm 0.28 \mathrm{Bb}$ & $0.37 \pm 0.02 \mathrm{Bbc}$ & $0.28 \pm 0.01 \mathrm{Bbc}$ & $0.14 \pm 0.01 \mathrm{Bc}$ & $0.10 \pm 0.01 \mathrm{Ac}$ & $0.07 \pm 0.01 \mathrm{Bc}$ & $0.07 \pm 0.01 \mathrm{Bc}$ \\
\hline \multirow{2}{*}{$\begin{array}{l}\text { GC Fuji II LC } \\
\text { Capsule }\end{array}$} & $20 \mathrm{~s}, 1300 \mathrm{~mW} / \mathrm{cm}^{2}$ & $2.01 \pm 0.19 \mathrm{Aa}$ & $0.25 \pm 0.02 \mathrm{Ab}$ & $0.09 \pm 0.01 \mathrm{Abc}$ & $0.12 \pm 0.04 \mathrm{Abc}$ & $0.09 \pm 0.02 \mathrm{Abc}$ & $0.03 \pm 0.00 \mathrm{Ac}$ & $0.02 \pm 0.00 \mathrm{Ac}$ & $0.01 \pm 0.00 \mathrm{Ac}$ \\
\hline & $5 \mathrm{~s}, 3000 \mathrm{~mW} / \mathrm{cm}^{2}$ & $2.62 \pm 0.25 \mathrm{Ba}$ & $0.46 \pm 0.03 \mathrm{Bb}$ & $0.34 \pm 0.12 \mathrm{Bb}$ & $0.15 \pm 0.02 \mathrm{Ac}$ & $0.13 \pm 0.04 \mathrm{Ac}$ & $0.04 \pm 0.01 \mathrm{Ac}$ & $0.02 \pm 0.00 \mathrm{Ac}$ & $0.01 \pm 0.00 \mathrm{Ac}$ \\
\hline & $\begin{array}{l}\text { The avera } \\
\text { contained } \\
\text { could not } \\
\text { row of eac } \\
\text { extraction }\end{array}$ & $\begin{array}{l}\text { aily release was c } \\
\text { released between } \\
\text { uantified at all, i.e. } \\
\text { ll. Different upper } \\
\text { es (in rows). }\end{array}$ & $\begin{array}{l}\text { ulated by dividin } \\
\text { ays } 10 \text { and } 16 \text {, so th } \\
\text { hat the concentrati } \\
\text { se letters indicate }\end{array}$ & $\begin{array}{l}\text { e amount of BPA } \\
\text { traction time was } \\
\text { f BPA in the extra } \\
\text { nnificant difference }\end{array}$ & $\begin{array}{l}\text { ne extract by the ex } \\
\text { ays. Zero values ind } \\
\text { as lower than the I } \\
\text { tween the polymer }\end{array}$ & $\begin{array}{l}\text { ction time. For exa } \\
\text { te either that the ca } \\
\text { Q. Significant diffe } \\
\text { ion conditions; dif }\end{array}$ & $\begin{array}{l}\text { le, the extract colle } \\
\text { ated daily release } \\
\text { ces between group } \\
\text { nt lowercase letter }\end{array}$ & $\begin{array}{l}\mathrm{d} 16 \text { days after } \mathrm{sp} \\
\text { eased below } 0.005 \\
\text { e indicated using } 1 \\
\text { dicate significant }\end{array}$ & $\begin{array}{l}\text { nen preparation } \\
/ \mathrm{g} / \text { day or that it } \\
\text { ers in the second } \\
\text { erences between }\end{array}$ \\
\hline
\end{tabular}


Table 3. Average daily release of BPA in methanol per gram of material (mean $\pm \mathrm{SD}$ in $\mathrm{ng} / \mathrm{g} /$ day).

\begin{tabular}{|c|c|c|c|c|c|c|c|c|c|}
\hline Material & $\begin{array}{l}\text { Polymer. } \\
\text { Conditions }\end{array}$ & 1 Day (Day 1) & $\begin{array}{l}4 \text { Days } \\
\text { (Days 2-4) }\end{array}$ & $\begin{array}{l}9 \text { Days } \\
\text { (Days 5-9) }\end{array}$ & $\begin{array}{l}16 \text { Days } \\
\text { (Days 10-16) }\end{array}$ & $\begin{array}{c}35 \text { Days } \\
\text { (Days 17-35) }\end{array}$ & $\begin{array}{c}65 \text { Days } \\
\text { (Days 36-65) }\end{array}$ & $\begin{array}{c}130 \text { Days } \\
\text { (Days 66-130) }\end{array}$ & $\begin{array}{l}260 \text { Days } \\
\text { (Days } \\
130-260)\end{array}$ \\
\hline \multirow{2}{*}{$\begin{array}{l}\text { Charisma } \\
\text { Classic }\end{array}$} & $20 \mathrm{~s}, 1300 \mathrm{~mW} / \mathrm{cm}^{2}$ & $9.01 \pm 1.23 \mathrm{Aa}$ & $2.36 \pm 0.19 \mathrm{Ab}$ & $1.57 \pm 0.17 \mathrm{Ac}$ & $1.90 \pm 0.44 \mathrm{Abc}$ & $1.54 \pm 0.07$ Acd & $1.03 \pm 0.07 \mathrm{Ad}$ & $0.78 \pm 0.06 \mathrm{Be}$ & $0.48 \pm 0.02 \mathrm{Bf}$ \\
\hline & $5 \mathrm{~s}, 3000 \mathrm{~mW} / \mathrm{cm}^{2}$ & $15.7 \pm 4.56 \mathrm{Ba}$ & $2.51 \pm 0.24 \mathrm{Ab}$ & $1.93 \pm 0.07 \mathrm{Abc}$ & $1.84 \pm 0.34 \mathrm{Abc}$ & $1.54 \pm 0.25 \mathrm{Acd}$ & $1.03 \pm 0.17 \mathrm{Ad}$ & $0.50 \pm 0.03 \mathrm{Ae}$ & $0.24 \pm 0.02 \mathrm{Af}$ \\
\hline \multirow{2}{*}{$\begin{array}{l}\text { Filtek Ultimate } \\
\text { Universal } \\
\text { Restorative }\end{array}$} & $20 \mathrm{~s}, 1300 \mathrm{~mW} / \mathrm{cm}^{2}$ & $15.0 \pm 0.64 \mathrm{Aa}$ & $1.66 \pm 0.22 \mathrm{Ab}$ & $1.21 \pm 0.10 \mathrm{Abc}$ & $1.12 \pm 0.02 \mathrm{Ac}$ & $1.14 \pm 0.04 \mathrm{Ac}$ & $1.82 \pm 0.04 \mathrm{Ab}$ & $1.09 \pm 0.10 \mathrm{Ac}$ & $0.88 \pm 0.06 \mathrm{Ac}$ \\
\hline & $5 \mathrm{~s}, 3000 \mathrm{~mW} / \mathrm{cm}^{2}$ & $25.6 \pm 7.03 \mathrm{Ba}$ & $2.61 \pm 0.52 \mathrm{Ab}$ & $1.68 \pm 0.31 \mathrm{Abc}$ & $1.40 \pm 0.30 \mathrm{Ac}$ & $1.99 \pm 0.04 \mathrm{Bb}$ & $2.12 \pm 0.19 \mathrm{Ab}$ & $1.21 \pm 0.05 \mathrm{Ac}$ & $0.94 \pm 0.03 \mathrm{Ad}$ \\
\hline \multirow{2}{*}{$\begin{array}{l}\text { Charisma } \\
\text { Diamond }\end{array}$} & $20 \mathrm{~s}, 1300 \mathrm{~mW} / \mathrm{cm}^{2}$ & $1.28 \pm 0.29 \mathrm{Aa}$ & $0.28 \pm 0.03 \mathrm{Ab}$ & $0.18 \pm 0.01 \mathrm{Ab}$ & $0.09 \pm 0.02 \mathrm{Ab}$ & $0.06 \pm 0.03 \mathrm{Ab}$ & $0.03 \pm 0.01 \mathrm{Ab}$ & $0.03 \pm 0.01 \mathrm{Ab}$ & $0.01 \pm 0.00 \mathrm{Ab}$ \\
\hline & $5 \mathrm{~s}, 3000 \mathrm{~mW} / \mathrm{cm}^{2}$ & $2.50 \pm 1.74 \mathrm{Ba}$ & $0.41 \pm 0.31 \mathrm{Ab}$ & $0.06 \pm 0.02 \mathrm{Ab}$ & $0.22 \pm 0.08 \mathrm{Ab}$ & $0.03 \pm 0.02 \mathrm{Ab}$ & $0.02 \pm 0.01 \mathrm{Ab}$ & $0.01 \pm 0.01 \mathrm{Ab}$ & $0.01 \pm 0.00 \mathrm{Ab}$ \\
\hline \multirow{2}{*}{ Admira Fusion } & $20 \mathrm{~s}, 1300 \mathrm{~mW} / \mathrm{cm}^{2}$ & $1.65 \pm 0.29 \mathrm{Aa}$ & $0.48 \pm 0.43 \mathrm{Ab}$ & $0.09 \pm 0.06 \mathrm{Ab}$ & $0.09 \pm 0.02 \mathrm{Ab}$ & $0.02 \pm 0.01 \mathrm{Ab}$ & $0.01 \pm 0.00 \mathrm{Ab}$ & $0.01 \pm 0.00 \mathrm{Ab}$ & $0.00 \pm 0.00 \mathrm{Ab}$ \\
\hline & $5 \mathrm{~s}, 3000 \mathrm{~mW} / \mathrm{cm}^{2}$ & $1.35 \pm 0.78 \mathrm{Aa}$ & $0.26 \pm 0.10 \mathrm{Ab}$ & $0.12 \pm 0.12 \mathrm{Ab}$ & $0.15 \pm 0.09 \mathrm{Ab}$ & $0.01 \pm 0.01 \mathrm{Ab}$ & $0.00 \pm 0.00 \mathrm{Ab}$ & $0.00 \pm 0.00 \mathrm{Ab}$ & $0.00 \pm 0.00 \mathrm{Ab}$ \\
\hline \multirow{2}{*}{$\begin{array}{c}\text { Photac Fil Quick } \\
\text { Aplicap }\end{array}$} & $20 \mathrm{~s}, 1300 \mathrm{~mW} / \mathrm{cm}^{2}$ & $3.71 \pm 0.47 \mathrm{Aa}$ & $0.56 \pm 0.12 \mathrm{Abc}$ & $0.57 \pm 0.03 \mathrm{Abc}$ & $0.43 \pm 0.07 \mathrm{Ac}$ & $0.45 \pm 0.02 \mathrm{Ac}$ & $0.57 \pm 0.06 \mathrm{Abc}$ & $0.87 \pm 0.21 \mathrm{Ab}$ & $0.83 \pm 0.01 \mathrm{Bb}$ \\
\hline & $5 \mathrm{~s}, 3000 \mathrm{~mW} / \mathrm{cm}^{2}$ & $9.27 \pm 2.44 \mathrm{Ba}$ & $1.11 \pm 0.26 \mathrm{Ab}$ & $0.62 \pm 0.04 \mathrm{Ac}$ & $0.60 \pm 0.05 \mathrm{Ac}$ & $0.70 \pm 0.07 \mathrm{Bc}$ & $0.94 \pm 0.06 \mathrm{Bbc}$ & $0.78 \pm 0.07 \mathrm{Abc}$ & $0.61 \pm 0.06 \mathrm{Ac}$ \\
\hline $\begin{array}{l}\text { GC Fuji II LC } \\
\text { Capsule }\end{array}$ & $5 \mathrm{~s}, 3000 \mathrm{~mW} / \mathrm{cm}^{2}$ & $2.91 \pm 0.41 \mathrm{Ba}$ & $1.46 \pm 0.19 \mathrm{Bb}$ & $0.61 \pm 0.09 \mathrm{Ac}$ & $0.38 \pm 0.08 \mathrm{Acd}$ & $0.17 \pm 0.02$ Ade & $0.08 \pm 0.01$ Ade & $0.05 \pm 0.01 \mathrm{Ae}$ & $0.03 \pm 0.01 \mathrm{Ae}$ \\
\hline
\end{tabular}


During the first day, significantly more BPA was released from specimens polymerized for $5 \mathrm{~s}$ at $3000 \mathrm{~mW} / \mathrm{cm}^{2}$ compared to those polymerized for $20 \mathrm{~s}$ at $1300 \mathrm{~mW} / \mathrm{cm}^{2}(p<0.05)$, except for PF in the artificial saliva and AF in methanol. However, at longer extraction times, the effect of polymerization conditions on the average daily release of BPA was not significant in most groups $(p>0.05)$. In terms of cumulative release (Table 4 , Figure 2 ), fast polymerization $\left(5 \mathrm{~s}, 3000 \mathrm{~mW} / \mathrm{cm}^{2}\right)$ led to a significantly higher release of BPA from RM-GICs in the artificial saliva and FU in methanol than standard polymerization did $\left(20 \mathrm{~s}, 1300 \mathrm{~mW} / \mathrm{cm}^{2}\right)$. On the contrary, CC released significantly higher amounts of BPA in methanol if polymerized for $20 \mathrm{~s}$, despite the tendency being initially opposite.

Table 4. Cumulative release of BPA per gram of material (mean $\pm \mathrm{SD}$ in $\mathrm{ng} / \mathrm{g}$ ).

\begin{tabular}{|c|c|c|c|c|c|c|c|}
\hline $\begin{array}{l}\text { Extraction } \\
\text { Medium }\end{array}$ & $\begin{array}{l}\text { Polymerization } \\
\text { Conditions }\end{array}$ & $\begin{array}{c}\text { Charisma } \\
\text { Classic }\end{array}$ & $\begin{array}{c}\text { Filtek } \\
\text { UltimateUniv. } \\
\text { Restorative }\end{array}$ & $\begin{array}{l}\text { Charisma } \\
\text { Diamond }\end{array}$ & Admira Fusion & $\begin{array}{c}\text { Photac Fil } \\
\text { Quick Aplicap }\end{array}$ & $\begin{array}{c}\text { GC Fuji II LC } \\
\text { Capsule }\end{array}$ \\
\hline \multirow{2}{*}{$\begin{array}{l}\text { Artificial } \\
\text { saliva }\end{array}$} & $\begin{array}{c}20 \mathrm{~s}, \\
1300 \mathrm{~mW} / \mathrm{cm}^{2}\end{array}$ & $143.7 \pm 6.0 \mathrm{Aa}$ & $180.4 \pm 39.1 \mathrm{Aa}$ & $2.09 \pm 0.48 \mathrm{Ab}$ & $2.54 \pm 0.95 \mathrm{Ab}$ & $14.1 \pm 1.7 \mathrm{Ac}$ & $8.84 \pm 1.28 \mathrm{Ad}$ \\
\hline & $\begin{array}{l}5 \mathrm{~s}, 3000 \\
\mathrm{~mW} / \mathrm{cm}^{2}\end{array}$ & $126.4 \pm 24.7 \mathrm{Aa}$ & $154.1 \pm 23.1 \mathrm{Aa}$ & $3.34 \pm 0.42 \mathrm{Ab}$ & $3.39 \pm 0.59 \mathrm{ABb}$ & $27.6 \pm 1.1 \mathrm{Bc}$ & $12.9 \pm 1.2 \mathrm{Bd}$ \\
\hline \multirow{2}{*}{ Methanol } & $\begin{array}{c}20 \mathrm{~s}, \\
1300 \mathrm{~mW} / \mathrm{cm}^{2}\end{array}$ & $213.1 \pm 4.90 \mathrm{Ba}$ & $299.0 \pm 18.2 \mathrm{Bb}$ & $7.98 \pm 0.46 \mathrm{Bc}$ & $5.11 \pm 1.65 \mathrm{Bd}$ & $206.1 \pm 17.0 \mathrm{Ca}$ & $22.7 \pm 3.0 \mathrm{Ce}$ \\
\hline & $\begin{array}{c}5 \mathrm{~s}, \\
3000 \mathrm{~mW} / \mathrm{cm}^{2}\end{array}$ & $170.8 \pm 19.0 \mathrm{Ca}$ & $358.0 \pm 16.3 \mathrm{Cb}$ & $8.53 \pm 0.82 \mathrm{Bc}$ & $4.40 \pm 1.03 \mathrm{ABd}$ & $194.3 \pm 7.8 \mathrm{Ca}$ & $25.4 \pm 2.2 \mathrm{Ce}$ \\
\hline
\end{tabular}

Cumulative values equal to the total amounts of BPA released from each material over the entire period of 260 days. Significant differences between groups are indicated using letters in the second row of each cell. Different uppercase letters indicate significant differences within each column; different lowercase letters indicate significant differences between the tested materials (in rows).

The cumulative data also showed that significantly more BPA was released in methanol $(p<0.001)$. The effect of materials was strongly significant as well $(p<0.001)$. Conventional composites (FU, CC) released substantially more BPA than RM-GICs (PF, F2) $(p<0.001)$, although the cumulative amount of BPA released from PF was similar to CC in methanol $(p>0.05)$. The least BPA was released from the "BPA-free" composites (AF, CD) $(p<0.001)$, regardless of the extraction medium.

\subsection{Uptake of Extraction Media and Mass Loss}

The uptake of artificial saliva was not significantly affected by polymerization conditions $(p>0.05)$, but fast polymerization for $5 \mathrm{~s}$ significantly increased the uptake of methanol $(p<0.05)$ in all composites except for CD (Table 5). The mass loss in methanol was significantly higher if composites had been polymerized for $5 \mathrm{~s}(p<0.05)$, and a similar tendency was noted in the artificial saliva, but it was not significant $(p>0.05)$ (Table 6). Both the uptake of extraction media and mass loss were significantly higher in methanol $(p<0.001)$. As for the effect of material, FU exhibited the highest uptake of artificial saliva $(p<0.001)$ and the lowest uptake of methanol $(p<0.001)$, regardless of polymerization conditions. AF had the highest mass loss in the artificial saliva when polymerized for $20 \mathrm{~s}$, but there was no significant difference between the materials if they were polymerized for $5 \mathrm{~s}$. In methanol, the order of materials according to the mass loss was CC $>\mathrm{CD}>\mathrm{AF}>\mathrm{FU}(p<0.05)$. 


\section{Cumulative release of BPA}
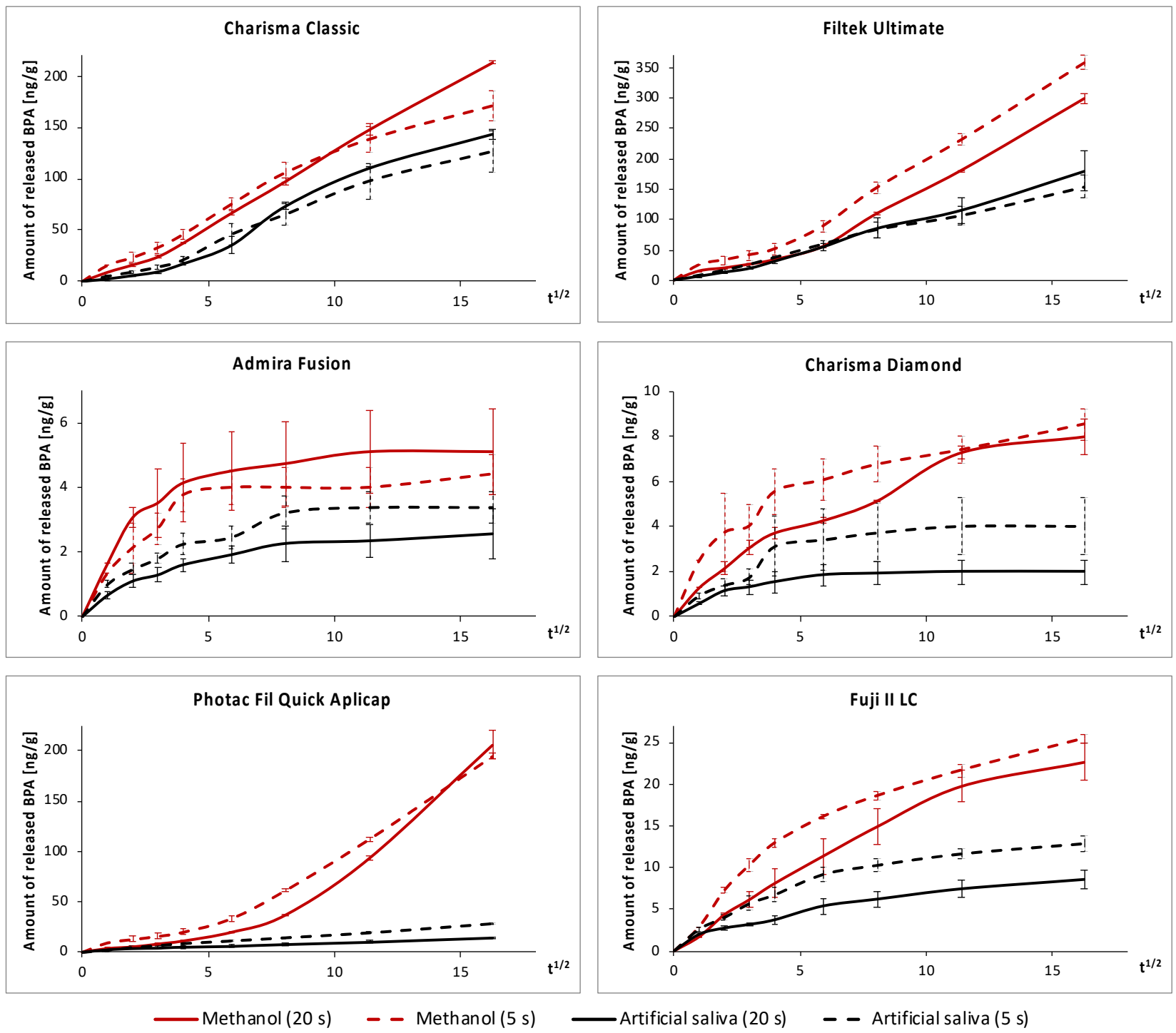

Figure 2. Cumulative amounts of BPA released in the artificial saliva (black) and methanol (red) plotted against the square root of time. The solid lines represent standard polymerization (20 s, $\left.1300 \mathrm{~mW} / \mathrm{cm}^{2}\right)$, whereas dashed lines represent fast polymerization $\left(5 \mathrm{~s}, 3000 \mathrm{~mW} / \mathrm{cm}^{2}\right)$.

Table 5. The uptake of extraction media by the tested composites (mean $\pm \mathrm{SD}$ in $\mathrm{wt} \%$ ).

\begin{tabular}{cccccc}
\hline $\begin{array}{c}\text { Extraction } \\
\text { Medium }\end{array}$ & $\begin{array}{c}\text { Polymerization } \\
\text { Conditions }\end{array}$ & $\begin{array}{c}\text { Charisma } \\
\text { Classic }\end{array}$ & $\begin{array}{c}\text { Filtek UltimateUniv. } \\
\text { Restorative }\end{array}$ & $\begin{array}{c}\text { Charisma } \\
\text { Diamond }\end{array}$ & Admira Fusion \\
\hline \multirow{2}{*}{ Artificial saliva } & $20 \mathrm{~s}, 1300 \mathrm{~mW} / \mathrm{cm}^{2}$ & $0.73 \pm 0.14 \mathrm{Aa}$ & $1.24 \pm 0.05 \mathrm{Ab}$ & $0.61 \pm 0.03 \mathrm{Aa}$ & $0.64 \pm 0.09 \mathrm{Aa}$ \\
\cline { 2 - 7 } & $5 \mathrm{~s}, 3000 \mathrm{~mW} / \mathrm{cm}^{2}$ & $0.68 \pm 0.17 \mathrm{Aa}$ & $1.27 \pm 0.01 \mathrm{Ab}$ & $0.52 \pm 0.02 \mathrm{Aa}$ & $0.72 \pm 0.004 \mathrm{Aa}$ \\
\hline \multirow{2}{*}{ Methanol } & $20 \mathrm{~s}, 1300 \mathrm{~mW} / \mathrm{cm}^{2}$ & $3.39 \pm 0.15 \mathrm{Ba}$ & $1.37 \pm 0.05 \mathrm{Bb}$ & $3.37 \pm 0.11 \mathrm{Ba}$ & $2.29 \pm 0.11 \mathrm{Bc}$ \\
\cline { 2 - 7 } & $5 \mathrm{~s}, 3000 \mathrm{~mW} / \mathrm{cm}^{2}$ & $3.89 \pm 0.17 \mathrm{Ca}$ & $1.62 \pm 0.17 \mathrm{Cb}$ & $3.48 \pm 0.01 \mathrm{Bc}$ & $2.49 \pm 0.07 \mathrm{Cd}$ \\
\hline
\end{tabular}

Significant differences between groups are indicated using letters in the second row of each cell. Different uppercase letters indicate significant differences within each column; different lowercase letters indicate significant differences between the tested materials (in rows). 
Table 6. The mass loss of the tested composites (mean $\pm \mathrm{SD}$ in $\mathrm{wt} \%$ ).

\begin{tabular}{cccccc}
\hline $\begin{array}{c}\text { Extraction } \\
\text { Medium }\end{array}$ & $\begin{array}{c}\text { Polymerization } \\
\text { Conditions }\end{array}$ & $\begin{array}{c}\text { Charisma } \\
\text { Classic }\end{array}$ & $\begin{array}{c}\text { Filtek UltimateUniv. } \\
\text { Restorative }\end{array}$ & $\begin{array}{c}\text { Charisma } \\
\text { Diamond }\end{array}$ & Admira Fusion \\
\hline \multirow{2}{*}{ Artificial saliva } & $20 \mathrm{~s}, 1300 \mathrm{~mW} / \mathrm{cm}^{2}$ & $0.05 \pm 0.02 \mathrm{Aa}$ & $0.04 \pm 0.01 \mathrm{Aa}$ & $0.06 \pm 0.02 \mathrm{Aa}$ & $0.12 \pm 0.05 \mathrm{Ab}$ \\
\cline { 2 - 7 } & $5 \mathrm{~s}, 3000 \mathrm{~mW} / \mathrm{cm}^{2}$ & $0.12 \pm 0.07 \mathrm{Aa}$ & $0.12 \pm 0.03 \mathrm{Aa}$ & $0.11 \pm 0.03 \mathrm{Aa}$ & $0.12 \pm 0.01 \mathrm{Aa}$ \\
\hline \multirow{2}{*}{ Methanol } & $20 \mathrm{~s}, 1300 \mathrm{~mW} / \mathrm{cm}^{2}$ & $2.27 \pm 0.10 \mathrm{Ba}$ & $0.38 \pm 0.06 \mathrm{Bb}$ & $2.04 \pm 0.08 \mathrm{Bc}$ & $1.13 \pm 0.08 \mathrm{Bd}$ \\
\cline { 2 - 7 } & $5 \mathrm{~s}, 3000 \mathrm{~mW} / \mathrm{cm}^{2}$ & $3.06 \pm 0.19 \mathrm{Ca}$ & $0.83 \pm 0.13 \mathrm{Cb}$ & $2.48 \pm 0.05 \mathrm{Cc}$ & $1.35 \pm 0.08 \mathrm{Cd}$ \\
\hline
\end{tabular}

Significant differences between groups are indicated using letters in the second row of each cell. Different uppercase letters indicate significant differences within each column; different lowercase letters indicate significant differences between the tested materials (in rows).

\section{Discussion}

As silver amalgam is gradually being phased out, restorative dentistry has relied on resin composites and GICs. However, they release unreacted monomers and other substances, which may have adverse effects on human health. Among them, the endocrinedisrupting effect of BPA has drawn particular attention. While the concentrations of BPA in saliva measured by Olea et al. in 1996 [16] were alarming, follow-up studies did not corroborate them [17-19]. Moreover, as resin-based materials underwent further development and the sensitivity of analytical methods increased [26-28], the reported amounts of released BPA decreased to levels far below the current limits. Nevertheless, as BPA may accumulate in adipose tissue $[1,3]$ and dental materials are listed among the sources of BPA [12], further research on various factors affecting its release is necessary.

The release of various substances from resin composites and RM-GICs is directly affected by the quality of their polymerization [22], which depends on the radiant exitance of the polymerization lamp and irradiation time. As the radiant exitance of modern highpower polymerization lamps may surpass $3000 \mathrm{~mW} / \mathrm{cm}^{2}$, it allows for the shortening of irradiation time down to $5 \mathrm{~s}$ or even less. However, our previous study showed that fast polymerization for $5 \mathrm{~s}$ at $3000 \mathrm{~mW} / \mathrm{cm}^{2}$ was insufficient on the bottom surface of $2 \mathrm{~mm}$ thick composite specimens, as opposed to the standard polymerization for $20 \mathrm{~s}$ at $1300 \mathrm{~mW} / \mathrm{cm}^{2}$ [31]. Therefore, the effect of these polymerization conditions on the release of BPA was investigated in this study, as previous reports were inconclusive [23-25].

The results of this study revealed that fast polymerization led to a significantly higher release of BPA from most materials during the first day, but polymerization conditions had almost no influence on the cumulative amounts of BPA released over the whole extraction period, so the first null hypothesis was partially rejected. The first-day release of BPA was significantly higher compared to other extraction periods, which agrees with in vivo findings of increased BPA levels in saliva during the first hours after the application of composites or sealants [34-40]. Initially, BPA was probably extracted from the superficial layers with a lower degree of conversion, mainly from the bottom surface of the specimens, because light energy is significantly attenuated while passing through the materials [31,41]. However, the release of BPA in the oral cavity might be lower, because the deepest layers are often not in contact with saliva. On the other hand, an oxygen-inhibited layer is present on the restoration surface and, if not removed by finishing and polishing, it could increase the release of BPA compared to this in vitro study where oxygen inhibition of polymerization was prevented by polymerizing the specimens through glass slides.

The rate of BPA release decreased significantly after the first day, which agrees with previous studies on the kinetics of BPA release $[25,42,43]$. This can be attributed to postirradiation polymerization, which improves the degree of conversion during the first hours/days after irradiation [31,44,45], and the fact that BPA had already been released from the superficial layers. The decrease in BPA release gradually continued toward zero in most materials, and despite using a very sensitive UPLC-MS/MS method, "BPA-free" composites and RM-GICs soon reached levels close to or even below the LLOQ. At such low levels, the reliability of the analysis is decreased, and the experiment was therefore 
stopped after 260 days even though the release of BPA from CC and FU in both extraction media and PF in methanol was clearly still ongoing even at the end of the 260 day period (Figure 2). In Figure 1, it can be noticed that the gradual decrease in the average daily release was sometimes interrupted by a slight increase. While this could be an irrelevant deviation of the values, a similar phenomenon was observed in a recent study by De Nys et al. who investigated the long-term release of BPA from four resin composites [28]. In aqueous media, the increase could be caused by the hydrolytic degradation of the polymer [28], but further investigation would be necessary to identify the degradation mechanism in methanol.

The effect of polymerization conditions on the release of BPA was most pronounced on the first day. As the radiant exposure, i.e., total light energy delivered to fast-polymerized specimens, was lower, we presume that the lower polymerization degree of the bottom surface [31] contributed to the significantly higher release of BPA from fast-polymerized specimens. In the following periods, there were few significant differences in BPA release between fast and standard polymerization, and as the released amounts were very low, their clinical relevance is disputable. However, the mass loss of all tested composites was higher if they were polymerized for $5 \mathrm{~s}$ - significantly in methanol and nonsignificantly in the artificial saliva. This indicates that fast polymerization results in a significantly higher release of various components, presumably due to the lower degree of conversion [22]. In addition, it was revealed that fast polymerization significantly increased the uptake of methanol in all composites except for $\mathrm{CD}$, which also supports this conclusion.

The cumulative data showed that regardless of polymerization conditions, significantly more BPA was released in methanol than in the artificial saliva. Organic alcohols are used to simulate the worst-case scenario of BPA release from resin-based materials, because they are better solvents of methacrylates than water. Consequently, their penetration into hydrophobic composites is faster, and they extract higher amounts of BPA than water or artificial saliva [22]. However, the release of BPA also depends on the monomers used, their purity, and other factors. This was confirmed by the present study, as BPA release was significantly affected by material type, which led to the rejection of the second null hypothesis. Conventional composites released significantly more BPA than other tested materials, because they contain more BPA-based monomers. While Bis-GMA is contained in both tested conventional composites, FU also contains the more hydrophilic Bis-EMA that could contribute to the fact that FU released the highest amounts of BPA and had the highest uptake of artificial saliva. BPA-based monomers were not disclosed in the material safety data sheets of RM-GICs and "BPA-free" composites, but they were identified in extracts of RM-GICs by previous studies $[29,30]$. The presence of BPA-based monomers and the easier diffusion of extraction media into the structure of RM-GICs presumably caused them to release significantly higher amounts of BPA than "BPA-free" composites. The release of BPA from "BPA-free" composites was slightly surprising, but as only trace amounts were released, it was probably a result of contamination during the manufacturing process.

In comparison with literature data, the amounts of BPA released from conventional composites were similar to the results of other studies using UPLC-MS/MS methods $[27,28]$ but substantially lower than those measured using less sensitive methods $[19,20]$. To our knowledge, the release of BPA from "BPA-free" composites and RM-GICs was not investigated by other authors. However, regardless of the material type, the amounts of BPA released from the tested materials seem to be a negligible contribution to daily exposure and significantly below the TDI of $4 \mu \mathrm{g} / \mathrm{kg}$ b.w. In the worst-case scenario, i.e., the release from $\mathrm{FU}$ polymerized for $5 \mathrm{~s}$ in methanol during the first day, the exposure from $1 \mathrm{~g}$ of the material would equal $0.009 \%$ of the TDI for a $70 \mathrm{~kg}$ adult and $0.03 \%$ of the TDI for a $20 \mathrm{~kg}$ child. It should also be noted that $1 \mathrm{~g}$ corresponds to several fillings. Alternatively, when calculated per surface area as advocated by De Nys et al. [28], the exposure from a single crown would equal $0.002-0.004 \%$ of the adult's TDI and $0.008-0.014 \%$ of the child's TDI. In the improbable case of a full-mouth reconstruction with direct composite crowns, 
the $70 \mathrm{~kg}$ adult would be exposed to $0.09 \%$ of the TDI, and it must be remembered that the release decreased significantly after the first day.

However, in vitro studies need to be interpreted prudently, because intraoral conditions could affect the release of BPA through factors that are difficult to simulate in vitro, such as the continuous flow of saliva, presence of bacteria and various enzymes, mechanical loading, and changes in temperature and $\mathrm{pH}$. It can also be seen as a limitation that the differences between specimen preparation in vitro and actual filling placement might influence the measured values. Therefore, future studies should investigate the release of BPA in vivo and its implications on human health, as there are many uncertainties especially regarding low-dose adverse effects [8-11] and the accumulation of BPA [1,3]. The EFSA is currently re-evaluating the evidence of a potential BPA hazard, and the current TDI might change, depending on the updated assessment that is soon to be released. Further research should also be aimed at the development of alternative monomers without a BPA structure.

\section{Conclusions}

Within the limitations of this in vitro study, it can be concluded that fast polymerization significantly increased the initial release of BPA from dental composites and RM-GICs. However, the amounts of BPA released from these materials were substantially lower than the current limits, so they could be considered as a negligible contribution to the daily exposure. On the other hand, no exposure should be dismissed as safe, because the effects of BPA on human health have not been fully clarified to date.

Author Contributions: Conceptualization, M.D. and P.B.; methodology, A.T., M.S., M.D. and P.B.; validation, M.S. and M.D.; formal analysis, A.T. and P.B.; investigation, A.T., M.S., R.V. and A.R.; resources, M.S., R.V. and A.R.; writing-original draft preparation, A.T.; writing-review and editing, M.S., R.V., A.R., M.D. and P.B.; visualization, A.T.; supervision, M.D. and P.B.; project administration, A.T.; funding acquisition, M.S., R.V., A.R., M.D. and P.B. All authors have read and agreed to the published version of the manuscript.

Funding: This research was funded by Charles University (grant Progres Q29/1LF) and MH CZDRO (Institute of Endocrinology, 00023761).

Data Availability Statement: Data available on request from the corresponding author.

Conflicts of Interest: The authors declare no conflict of interest.

\section{References}

1. Vandenberg, L.N.; Maffini, M.V.; Sonnenschein, C.; Rubin, B.S.; Soto, A.M. Bisphenol-A and the great divide: A review of controversies in the field of endocrine disruption. Endocr. Rev. 2009, 30, 75-95. [CrossRef] [PubMed]

2. Kolatorova Sosvorova, L.; Chlupacova, T.; Vitku, J.; Vlk, M.; Heracek, J.; Starka, L.; Saman, D.; Simkova, M.; Hampl, R. Determination of selected bisphenols, parabens and estrogens in human plasma using LC-MS/MS. Talanta 2017, 174, 21-28. [CrossRef]

3. Diamanti-Kandarakis, E.; Bourguignon, J.-P.; Giudice, L.C.; Hauser, R.; Prins, G.S.; Soto, A.M.; Zoeller, R.T.; Gore, A.C. Endocrinedisrupting chemicals: An Endocrine Society scientific statement. Endocr. Rev. 2009, 30, 293-342. [CrossRef] [PubMed]

4. Rochester, J.R. Bisphenol A and human health: A review of the literature. Reprod. Toxicol. 2013, 42, 132-155. [CrossRef] [PubMed]

5. Kolatorova, L.; Duskova, M.; Vitku, J.; Starka, L. Prenatal exposure to bisphenols and parabens and impacts on human physiology. Physiol. Res. 2017, 66, S305-S315. [CrossRef]

6. The United States Food and Drug Administration. Bisphenol A (BPA): Use in Food Contact Application. Available online: https:/ / www.fda.gov/food/food-additives-petitions/bisphenol-bpa-use-food-contact-application (accessed on 22 July 2021).

7. EFSA Panel on Food Contact Materials, Enzymes, Flavourings and Processing Aids. Scientific opinion on the risks to public health related to the presence of bisphenol A (BPA) in foodstuffs. EFSA J. 2015, 13, 3978. [CrossRef]

8. Vandenberg, L.N.; Colborn, T.; Hayes, T.B.; Heindel, J.J.; Jacobs, D.R., Jr.; Lee, D.H.; Shioda, T.; Soto, A.M.; vom Saal, F.S.; Welshons, W.V.; et al. Hormones and endocrine-disrupting chemicals: Low-dose effects and nonmonotonic dose responses. Endocr. Rev. 2012, 33, 378-455. [CrossRef]

9. Birnbaum, L.S. Environmental chemicals: Evaluating low-dose effects. Environ. Health Perspect 2012, 120, a143-a144. [CrossRef]

10. vom Saal, F.S.; Hughes, C. An extensive new literature concerning low-dose effects of bisphenol A shows the need for a new risk assessment. Environ. Health Perspect 2005, 113, 926-933. [CrossRef] 
11. Vogel, S.A. The politics of plastics: The making and unmaking of bisphenol a "safety". Am. J. Public Health 2009, 99 (Suppl. S3), S559-S566. [CrossRef]

12. Geens, T.; Aerts, D.; Berthot, C.; Bourguignon, J.-P.; Goeyens, L.; Lecomte, P.; Maghuin-Rogister, G.; Pironnet, A.-M.; Pussemier, L.; Scippo, M.-L.; et al. A review of dietary and non-dietary exposure to bisphenol-A. Food Chem. Toxicol. 2012, 50, 3725-3740. [CrossRef]

13. Fleisch, A.F.; Sheffield, P.E.; Chinn, C.; Edelstein, B.L.; Landrigan, P.J. Bisphenol A and related compounds in dental materials. Pediatrics 2010, 126, 760-768. [CrossRef] [PubMed]

14. De Nys, S.; Duca, R.C.; Vervliet, P.; Covaci, A.; Boonen, I.; Elskens, M.; Vanoirbeek, J.; Godderis, L.; Van Meerbeek, B.; Van Landuyt, K.L. Bisphenol A as degradation product of monomers used in resin-based dental materials. Dent. Mater. 2021, 37, 1020-1029. [CrossRef]

15. Soderholm, K.J.; Mariotti, A. BIS-GMA-based resins in dentistry: Are they safe? J. Am. Dent. Assoc. 1999, 130, 201-209. [CrossRef] [PubMed]

16. Olea, N.; Pulgar, R.; Pérez, P.; Olea-Serrano, F.; Rivas, A.; Novillo-Fertrell, A.; Pedraza, V.; Soto, A.M.; Sonnenschein, C. Estrogenicity of resin-based composites and sealants used in dentistry. Environ. Health Perspect 1996, 104, 298-305. [CrossRef]

17. Habib, C.M.; Kugel, G. Estrogenicity of resin-based composites and sealants in dentistry. Environ. Health Perspect 1996, $104,808$. [CrossRef]

18. Imai, Y. Comments on "Determination of bisphenol A and related aromatic compounds released from bis-GMA-based composites and sealants by high performance liquid chromatography". Environ. Health Perspect 2000, 108, a545-546. [CrossRef] [PubMed]

19. Kechagias, K.; Anastasaki, P.; Kyriakidou, M.; Dedi, K.D. Bisphenol A in dentistry. Eur. J. Prosthodont. Restor. Dent. 2020, 28 , 3-9. [CrossRef] [PubMed]

20. Löfroth, M.; Ghasemimehr, M.; Falk, A.; Vult von Steyern, P. Bisphenol A in dental materials—Existence, leakage and biological effects. Heliyon 2019, 5, e01711. [CrossRef]

21. Testai, E.; Epstein, M.; Emri, I.; Hartemann, P.; Hoet, P.; Leitgeb, N.; Martínez Martinez, L.; Proykova, A.; Rizzo, L.; RodriguezFarré, E.; et al. The safety of the use of bisphenol A in medical devices. Regul. Toxicol. Pharmacol. 2016, 79, 106-107. [CrossRef]

22. Van Landuyt, K.L.; Nawrot, T.; Geebelen, B.; De Munck, J.; Snauwaert, J.; Yoshihara, K.; Scheers, H.; Godderis, L.; Hoet, P.; Van Meerbeek, B. How much do resin-based dental materials release? A meta-analytical approach. Dent. Mater. 2011, 27, 723-747. [CrossRef] [PubMed]

23. Manabe, A.; Kaneko, S.; Numazawa, S.; Itoh, K.; Inoue, M.; Hisamitsu, H.; Sasa, R.; Yoshida, T. Detection of bisphenol-A in dental materials by gas chromatography-mass spectrometry. Dent. Mater. J. 2000, 19, 75-86. [CrossRef] [PubMed]

24. Kwon, H.J.; Oh, Y.J.; Jang, J.H.; Park, J.E.; Hwang, K.S.; Park, Y.D. The effect of polymerization conditions on the amounts of unreacted monomer and bisphenol A in dental composite resins. Dent. Mater. J. 2015, 34, 327-335. [CrossRef] [PubMed]

25. Polydorou, O.; König, A.; Hellwig, E.; Kümmerer, K. Long-term release of monomers from modern dental-composite materials. Eur. J. Oral Sci. 2009, 117, 68-75. [CrossRef]

26. Simkova, M.; Tichy, A.; Duskova, M.; Bradna, P. Dental composites-A low-dose source of bisphenol A? Phys. Res. 2020, 69, S295-S304. [CrossRef]

27. Becher, R.; Wellendorf, H.; Sakhi, A.K.; Samuelsen, J.T.; Thomsen, C.; Bølling, A.K.; Kopperud, H.M. Presence and leaching of bisphenol A (BPA) from dental materials. Acta Biomater. Odontol. Scand. 2018, 4, 56-62. [CrossRef]

28. De Nys, S.; Putzeys, E.; Duca, R.C.; Vervliet, P.; Covaci, A.; Boonen, I.; Elskens, M.; Vanoirbeek, J.; Godderis, L.; Van Meerbeek, B.; et al. Long-term elution of bisphenol A from dental composites. Dent. Mater. 2021, 37, 1561-1568. [CrossRef] [PubMed]

29. Rogalewicz, R.; Batko, K.; Voelkel, A. Identification of organic extractables from commercial resin-modified glass-ionomers using HPLC-MS. J. Environ. Monit. 2006, 8, 750-758. [CrossRef]

30. Mazzaoui, S.A.; Burrow, M.F.; Tyas, M.J.; Rooney, F.R.; Capon, R.J. Long-term quantification of the release of monomers from dental resin composites and a resin-modified glass ionomer cement. J. Biomed. Mater. Res. 2002, 63, 299-305. [CrossRef]

31. Tichy, A.; Bradna, P. Applicability of exposure reciprocity law for fast polymerization of restorative composites containing various photoinitiating systems. Oper Dent. 2021, 46, 406-418. [CrossRef]

32. Bradna, P.; Vrbova, R.; Fialova, V.; Housova, D.; Gojisova, E. Formation of protective deposits by anti-erosive toothpastes-A microscopic study on enamel with artificial defects. Scanning 2016, 38, 380-388. [CrossRef]

33. Vitku, J.; Chlupacova, T.; Sosvorova, L.; Hampl, R.; Hill, M.; Heracek, J.; Bicikova, M.; Starka, L. Development and validation of LC-MS/MS method for quantification of bisphenol A and estrogens in human plasma and seminal fluid. Talanta 2015, 140, 62-67. [CrossRef]

34. Arenholt-Bindslev, D.; Breinholt, V.; Preiss, A.; Schmalz, G. Time-related bisphenol-A content and estrogenic activity in saliva samples collected in relation to placement of fissure sealants. Clin. Oral Investig. 1999, 3, 120-125. [CrossRef] [PubMed]

35. Fung, E.Y.K.; Ewoldsen, N.O.; St. Germain, H.A.; Marx, D.B.; Miaw, C.-L.; Siew, C.; Chou, H.-N.; Gruninger, S.E.; Meyer, D.M. Pharmacokinetics of bisphenol A released from a dental sealant. J. Am. Dent. Assoc. 2000, 131, 51-58. [CrossRef] [PubMed]

36. Lee, J.-H.; Yi, S.-K.; Kim, S.-Y.; Kim, J.-S.; Son, S.-A.; Jeong, S.-H.; Kim, J.-B. Salivary bisphenol A levels and their association with composite resin restoration. Chemosphere 2017, 172, 46-51. [CrossRef]

37. Zimmerman-Downs, J.M.; Shuman, D.; Stull, S.C.; Ratzlaff, R.E. Bisphenol A blood and saliva levels prior to and after dental sealant placement in adults. J. Dent. Hyg. 2010, 84, 145-150. 
38. Sasaki, N.; Okuda, K.; Kato, T.; Kakishima, H.; Okuma, H.; Abe, K.; Tachino, H.; Tuchida, K.; Kubono, K. Salivary bisphenol-A levels detected by ELISA after restoration with composite resin. J. Mater. Sci. Mater. Med. 2005, 16, 297-300. [CrossRef]

39. Joskow, R.; Barr, D.B.; Barr, J.R.; Calafat, A.M.; Needham, L.L.; Rubin, C. Exposure to bisphenol A from bis-glycidyl dimethacrylatebased dental sealants. J. Am. Dent. Assoc. 2006, 137, 353-362. [CrossRef]

40. Berge, T.L.L.; Lygre, G.B.; Lie, S.A.; Lindh, C.H.; Bjorkman, L. Bisphenol A in human saliva and urine before and after treatment with dental polymer-based restorative materials. Eur. J. Oral Sci. 2019, 127, 435-444. [CrossRef] [PubMed]

41. Price, R.B.; Murphy, D.G.; Derand, T. Light energy transmission through cured resin composite and human dentin. Quintessence Int. 2000, 31, 659-667.

42. De Nys, S.; Putzeys, E.; Vervliet, P.; Covaci, A.; Boonen, I.; Elskens, M.; Vanoirbeek, J.; Godderis, L.; Van Meerbeek, B.; Van Landuyt, K.L.; et al. A novel high sensitivity UPLC-MS/MS method for the evaluation of bisphenol A leaching from dental materials. Sci. Rep. 2018, 8, 6981. [CrossRef] [PubMed]

43. Imai, Y.; Komabayashi, T. Elution of bisphenol A from composite resin: A model experiment. Dent. Mater. J. 2000, 19, $133-138$. [CrossRef] [PubMed]

44. Pilo, R.; Cardash, H.S. Post-irradiation polymerization of different anterior and posterior visible light-activated resin composites. Dent. Mater. 1992, 8, 299-304. [CrossRef]

45. Leung, R.L.; Fan, P.L.; Johnston, W.M. Post-irradiation polymerization of visible light-activated composite resin. J. Dent. Res. 1983, 62, 363-365. [CrossRef] 